\title{
Travel Behavior Modeling: Taxonomy, Challenges, and Opportunities
}

\author{
Aman Sharma ${ }^{1}$, Abdullah Gani ${ }^{2}$, David Asirvatham ${ }^{3}$, Riyath Ahmed ${ }^{4}$, \\ Muzaffar Hamzah ${ }^{5}$, Mohammad Fadhli Asli ${ }^{6}$ \\ Faculty of Innovation and Technology, Taylor's University Malaysia ${ }^{1,3,4}$ \\ Faculty of Computing and Informatics, Universiti Malaysia Sabah, Malaysia ${ }^{2,5,6}$
}

\begin{abstract}
Personal daily movement patterns have a longitudinal impact on the individual's decision-making in traveling. Recent observation on human travel raises concerns on the impact of travel behavior changes on many aspects. Many travel-related aspects like traffic congestion management and effective land-use were significantly affected by travel behavior changes. Existing travel behavior modeling (TBM) were focusing on assessing traffic trends and generate improvement insights for urban planning, infrastructure investment, and policymaking. However, literature indicates limited discussions on recent TBM adaptation towards future technological advances like the integration of autonomous vehicles and intelligent traveling. This survey paper aims to provide overview insights on recent advances of TBM including notable classifications, emerging challenges, and rising opportunities. In this survey, we reviewed and analyzed recently published works on TBM from high-quality publication sources. A taxonomy was devised based on notable characteristics of TBM to guide the classification and analysis of these works. The taxonomy classifies recent advances in TBM based on type of algorithms, applications, data sources, technologies, behavior analysis, and datasets. Furthermore, emerging research challenges and limitations encountered by recent TBM studies were characterized and discussed. Subsequently, this survey identified and highlights open issues and research opportunities arise from recent TBM advances for the future undertaking.
\end{abstract}

Keywords-Travel behavior; travel behavior modeling; prediction modeling; intelligent traveling

\section{INTRODUCTION}

Personal daily movement habit cumulatively influences the decision-making approach of the individual when traveling. Recent observation on human travels raises concerns on the impact of recent travel behavior changes towards many aspects including traffic management, transport planning, and decisionmaking. Researchers carried out travel behavior studies with the goal of understanding human travel behavior via analytical approach. The main goal of travel behavior studies is to generate translational travel behavior model for facilitating prediction analysis for travel planning insights. Therefore, Travel Behavior Modeling (TBM) is introduced as costefficient and timesaving approach for supporting that goal. Traffic management are severely affected by increasing traffic congestion occurrence due to human travel behavior. Recent observation pointed out the increases of inferior travel habit like slow driving style that further amplified by faulty road condition and crowded area. These behaviors and external factors eventually cause indirect losses on many aspects. Massive transport congestion in the US costed the economy with average wasted 5.5B work hours, more than 2.9B gallons of fuel, and more $\$ 100$ billion per year. China also faced similar problem with consequences of increasing annual travel cost that currently priced at $\$ 1,126$ per person [1]. Victoria Transport Policy Institute highlighted that traffic congestion is the leading cause of critical delay, high fuel consumption and economic wastage in the US. This issue has considerably contributed towards decline in trade and growth. This expense is expected to rise by 2020 to the extent of $\$ 121 \mathrm{~B}$ [2].

Future travel behavior is expected to change drastically soon due to mainstream popularity of autonomous vehicles and smart travel technologies. Increasing adaptation of autonomous vehicles eventually change how human travel, consequently impacting travel behavior norms and environment [3]. The use of autonomous vehicles increases travel mileage with reduced share of slowing traffics with other slow-mode public transports. These changes are already reflected in growth pattern in many dispersed urban cities. Eventually, autonomous vehicles reduce the need for private vehicles and provide extraneous conveniences for user despite its minor contribution towards parking congestion. A recent study reported the implications of substituting all private motor vehicles with shared autonomous vehicles (SAV) in Berlin, Germany [4]. Their findings indicates that the substitution is likely to decrease the number of cars that accommodates private travel demand by $91 \%$. In addition, the number of vehicles that accommodates travel demand in Lisbon are expected to decrease by $95 \%$ with the introduction of SAV [5]. Moreover, travel demand involving family-sharing private vehicles are also expected to significantly decrease due to redundant purpose [6]. However, such substitution in Atlantis, US only observe minor decrease of only $9.5 \%$ due to lower priority on accommodating individual travel demand. In addition, the association of travel behavior with socioeconomic (income, household composition) indicates land-use patterns that is leveraged to assess the mobility of individual traveler [7]. TBM is expected to play significant roles in realizing these future endeavors due to its direct role towards predicting traveler choices.

Many studies were conducted over the years in developing methods, algorithms, and models for TBM. Such examples of modeling are Short-term Travel Behavior Prediction [8], Intra-Household TBM [9], Sense Place TBM [10], Markov Travel Model [11], and Inferring Structural Models [12]. These modeling were built for facilitating prediction on travel demand and travel decision. Many TBM studies analyzes and diagnoses travel behavior pattern at individual level [13], [14], [15], [16]. Eventually, specific modeling for this scale were developed like Individual TBM [14], Regret-based Choice 
Model [13], Rethinking TBM [16] and Heterogeneous TBM [15]. With the upcoming changes from autonomous vehicles adaptation, land-use transposition, we can anticipate that TBM holds many potentials with lasting impact towards improving this aspect.

This survey aims to provide overview insights on recent advances of travel behavior modeling including emerging trends, challenges, and opportunities. Gaining latest insights on travel behavior modeling eventually contribute towards driving forward many sectors like transportation, tourism, and urban development. In this survey, recent published works on TBM from quality journals and established conferences indexed by Web of Science (WoS) were reviewed. A taxonomy was devised based on the survey to classify recent TBM advances based on many notable aspects. Furthermore, the problems and issues addressed by recent work were reviewed to derive the existing research challenges in TBM. Moreover, this survey analyzed the reported limitations and future works to highlight any persistent issues or opportunities in TBM for future studies. The main contributions of this survey paper are:

1) A taxonomy of travel behavior modeling that classifies TBM based on algorithms, applications, data collection techniques, technologies, travel behaviors, models, and datasets.

2) A summarization of emerging trends on research challenges and limitations in implementing TBM in present applications.

3) A summarization of open issues and opportunities from recent advances in TBM for future undertaking.

The content of this paper is organized as follows. Section 2 explains the fundamentals of travel behavior modeling including definitions, process, and terminologies. Section 3 introduces the devised taxonomy of travel behavior modeling based on the conducted survey including characterizations and instances of each classification. Section 4 then presents the summarization of identified research challenges and limitations from existing TBM. Section 5 then highlights the open issues and opportunities arises from recent TBM advances for future studies. Finally, Section 6 presents the conclusions for this paper.

\section{Travel Behavior Modeling}

This section introduces the fundamentals of travel behavior modeling including the underlying concepts, definitions, and working terminologies. The presented fundamentals offer general information on travel behavior modeling for understanding the remaining contents in this paper.

The origin of travel behavior modeling started by the introduction of behavioral modeling to systems theory and control theory in 1970s [17]. Behavior modeling addresses the conventional approaches' inconsistencies on state-space, transfers, and convolution representations. Eventually, behavior modeling is adapted in many event or activity analysis over the years including travel behavior analysis. Travel behavior analysis is important in supporting sustainable development in parallel with society growth and technological advances. Travel behavior studies are carried out to explore human travel behavior's impacts towards fuel consumption, environment, urban development, and citizen upbringing.

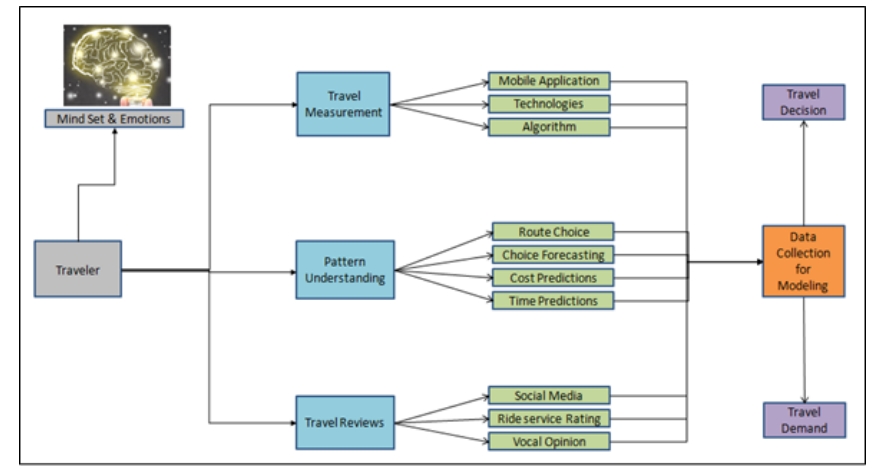

Fig. 1. Functional Model of Travel Behavior Modeling.

Travel behavior analysis starts by anticipating the mindset of traveler associated with travel measurement, pattern understating, and travel reviews. In general, TBM is utilized to process these variables to generate prediction on travel decision and travel demand. TBM learns user activities based on recorded travel experiences information like time, place, and mode of transportation. TBM then diagnose, understand, and response to the change of activities and subsequently predict future travel decision. Eventually, the generated prediction should accommodate travel habits and transportation in future travel based on the preferences and lifestyle of the user. Fig. 1 summarizes the framework of overall processes and related attributes in travel behavior modeling.

To help readers in understanding the remaining contents in this paper, we specify the working terminologies that are commonly used as follows:

- Algorithms: Processing operations or rule sets of the analysis models created by travel behavior modeling.

- Applications: Computer programs or software that implemented travel behavior modeling as core function with the purpose of facilitating travel behavior analysis.

- Models: Machine learning models created based on statistical or mathematical approach to extract and learn behavior patterns from travel datasets.

- Industries: Industrial domains, sectors, or fields of the applied travel behavior modeling.

\section{TAXonomy of Travel Behavior Modeling}

This section introduces the taxonomy on travel behavior modeling based on the conducted survey as illustrated in Fig. 2. This taxonomy classifies travel behavior modeling based on algorithms, applications, data sources, technologies, analysis, models, and testing datasets. Characterizations of each travel behavior modeling classification are presented as follows.

\section{A. Travel Behavior Modeling Algorithms}

Our taxonomy classifies the algorithms of recent TBM advances based on the algorithm objectives and approaches. The algorithms of recent TBM advances are classified and characterized as follows. 


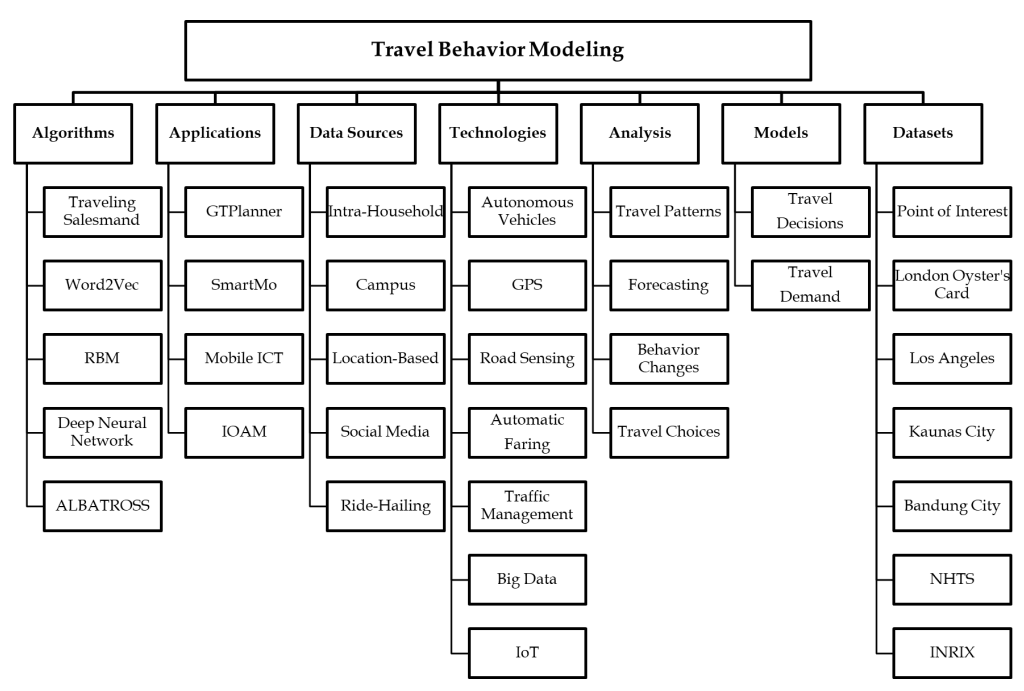

Fig. 2. Taxonomy of Travel Behavior Modeling.

1) Traveling Salesman: The crucial aspect in travel behavior modeling is the efficiency of behavior modeling optimization. Optimizing algorithm like Traveling Salesman are used in several instances such as benchmarking, performance comparison, algorithm selection, algorithm analysis, and algorithm comparison. Optimizing algorithm model is achieved using two ways: (1) fitting nonlinear curves with fixed semantics and (2) training artificial neural networks on benchmark results. A study demonstrated effective travel behavior modeling based on Traveling Salesman algorithm by using model optimization approach [18].

2) Word2Vec: Albeit the various methods available for analyzing travel demand prediction, automatic translation and word prediction are crucial in facilitating the analysis. The Word2vec algorithm allows the recalibration of PyTre packages on supporting automated translation and prediction via natural language processing (NLP). By leveraging natural language processing, performance measurement on testing or training dataset is carried out swiftly. Despite the swift advantages, the algorithm is limited by its stochastic nature that eventually requires implementation via deliberate experimental design. In addition, integrating model like Rethinking Travel Behavior (RTB) is viable in the algorithm development to support performance measurement. RTB model predicts the travel demand based on mode, trip purpose, education level, family type, and occupation. Furthermore, the model utilizes the same datasets throughout processing for consistency [16].

3) Restricted Boltzmann Machine: This algorithm enables consideration of information heterogeneity and variable correlations in processing that commonly occurs on large scale datasets. A recent study investigated on generative machine learning approaches by analyzing multiple discrete-continuous (MDC) travel behavior event from 293,330 travel data [19]. RBM-based algorithm performs significantly better in modeling behavior with larger datasets due to increased modeling accuracy and future forecasting. The study proposed a framework that recommends RGM-based algorithm implementation based on different instances of route choice, dynamic road pricing, and traffic simulation application.
4) Deep Neural Network: Taking advantages of the prowess of deep learning, researchers start to implement deep neural network model to predict travel choices pattern. A recent study proposed a deep neural network structure model that allows many additional structural settings like hidden layer structure, activation functions, number of training epochs, dropout ratio, and learning set ratio [20]. The proposed approach leverages deep learning capabilities to models traveler behavior using deep neural network structure.

5) ALBATROSS: Machine learning identifies that versatile application which is related to the ALBATROSS model using decision trees. Through this model, researchers can focus more on freight transport research, feature engineering, driver behavior, data extraction from digital media and the GPS trajectories that are focusing on transport research [21].

\section{B. Travel Behavior Modeling Applications}

In general, travel behavior modeling applications aim to support event pattern analysis on travel demand and planning. However, the applications comprise of different features depending on the travel sectors or the scale of users it covers. Instances of application and its distinct features are presented as follows.

1) GTPlanner: GTPlanner uses TBM for facilitating transportation system planning by generating travel insights based on user preferences and duration [22]. GTPlanner is an exemplary educational travel advisor by promoting the impact of individual traveling modes which consequently fostering quality travel behaviors. GTPlanner also features rudimentary trip planner that allows en route behavior changes tracking and generates latest insight prospects.

2) SmartMo: SmartMo is a mobile-based application for gathering traveler real-time information and generate real-time travel insights. SmartMo gathers and assess event information like pre-trip (mode of transportation and trip purpose), on-trip (start trip, route tracking and end trip), and post-trip(upload). Taking advantages of traveler event information, SmartMo 
enables real-time route checking, insights update, data autonomy, data security, and time efficiency for monitoring traveler behavior [23].

3) Mobile ICT: Nowadays, mobile devices like smartphone and tablets plays significant role in facilitating travel activities with travel planning and social networking features. Travel planning is simpler than ever before, when smart devices and applications have become mainstream in human culture. Planning tasks like browsing travel date, mode of travel, destination, and coordinating trip decisions are easily carried out via mobile devices. With mobile devices, travelers can fully utilize any online advantages to maximize the travel outcomes throughout the trip duration. Such instances of outcomes including increased count of places visited, social gatherings attended, and trips planned in groups [24]. Empowered by mobile computing, travel behavior and tourist business also change drastically over the years [25]. Travel behavior comprises 5 core phases: dreaming (reviews, blogs, travelers' experiences), planning (information and travel guidance), booking (flight, accommodation, bus, car, and tours), experiencing (on demand booking and maps) and sharing (friends, relatives, and social network) [26].

4) Interactive Online Accessbility Mapping (IOAM): Interactive web usability mapping facilitates many traveling purposes like commuting, school, healthcare, lodging, social activities, and entertainment. IOAM features four transportation modes: walking, biking, vehicle transit, and selected destination requirements. A recent study investigated the role of IOAM in facilitating users on understanding travel behavior. The study reported that participants with access to IOAM valued the tool in understanding residential location and travelrelated decision-making process [27].

\section{Travel Behavior Modeling Data Sources}

Working data for TBM are originated from various sources like social media, crowdsourcing, or service providers. Many TBM studies work with data generated using survey-based approach that utilizes user interviews and online questionnaires. Such instances of data sources that were found in the survey are related with intra-household, campus, location-based tool, social media, and ride-hailing services. The characterizations of each related data sources are discussed as follows.

1) Intra-Household: Modeling and forecasting traveler's time preference takes account on several individual aspects into consideration like demographics, situational, psychological, and sociological. For instance, predicting bus travel time preferences largely dependent on parameters of different modes and actors like bus drivers, other bus users, and other metro users [11]. Another instance is the modeling of individual travel behavior based on intra-household interactions via datasets of $67 \mathrm{~K}$ trips made by $3.5 \mathrm{~K}$ individuals [9]. In general, suitable data collection techniques for this type of TBM relies heavily on the individual familiar sense of the location. Another instance is the modeling of travel decision among users between two shopping centers in California via 719 survey data [10]. The utilized TBM assess movement patterns between two traveler vehicles based on two factors: energy conservation and environmental protection. Another instance of intra-household consideration in TBM is travel activity assessment for addressing fuel consumption, car uses, and gas emission in South India [28].

2) Campus: The goal of using TBM for modeling individual travel behavior in campus is to generate mapping on daily transport travel demand. For instance, Dalhousie University, Canada carried out a travel survey among its campus residents pertaining travel activities and environmental awareness. The survey gathered information from campus residents like resident's work, entertainment activities, social activities, formal education activities, and shopping activities [29]. In addition, many travel projects especially from European countries often generated their travel behavior datasets using GPS with volunteer participants from urban cities.

3) Location-Based Tool \& Services: The rapid advances of location-based services have turned location-based tool into a powerful data gathering and communication methods. For instance, a study observed travel check-in behavior from social media data by analyzing its location-based social network (LBSN) features [30]. Their findings reveal contrasting pattern of check-in behavior between weekday and weekend activities, with emergences of unique patterns exhibited by different gender. Their study demonstrated on how spatial pattern of checkin mapping surface density and smoothness are ascertained using kernel density estimation (KDE) associated ArcGIS.

4) Social Media: Social media has become the mainstream online interaction platform for discussing or sharing opinions and experiences on traveling. Social media usage on traveling topics holds myriads of information like patterns and reviews of past traveling events recorded by many travelers. Social media network like Facebook, Twitter, and Yelp can generate enriched travel movement behavior data via location-based sharing features [31]. In their study, Rashidi et al. reviewed many possible methods for analyzing travel-related social media data effectively [32]. They highlighted that the geotagging features are leveraged by city planners and business premises to understand the urban dynamic of their areas. Gaining insights on recent urban dynamic can help these entities to adapt instead of relying on outdated information from old records. Featuring services like check-in logs and hotspot, social media data helps researchers to understand the travel demand of visited locations. Location-based information from these services allows TBM to predict individual travel behavior with better accuracy and efficiency. However, limited access towards those social media data remains to be the main issue for data mining and natural language processing. Restricted data fetching indirectly reduces the quality of insights on travel behavior that are obtained from social media [33], [34]. Another instance is how a study implemented logistic regression model for analyzing Facebook check-in logs to predict future check-in patterns [35]. Their findings indicate how majority of Singaporeans adapted and modified their leisure travel behavior via social media influences. Another study highlighted the significant impacts of social media in travel itinerary planning and social sharing norm [36].

The advances of social media platform also amplify social media data usage generation, resulting massive collection of user interaction. There are several technical challenges in analyzing social media big data for understanding travel behavior like incompatible text parsing, data format inconsistencies, and hidden sentiments. For instance, Twitter social media 
data contains check-in logs enabled by geo-tagging features along with text posting information [37]. Travel studies can leverage the geo-tagged information to identify the relationship between conventional model of travel demands [38], [39]. The prospect of traffic incidents identification and verification via Twitter opens the possibilities for efficient and real-time data management. Moreover, Instagram allows location sharing and posting of travel experiences via pictorial, posts, and videos [40]. Other social media platform like Foursquare also records individual travel behavior information based on check-in logs, number of visits, and activity patterns [41]. A movement behavior study on bike-sharing services in Washington used probabilistic models and analysis to understand bike user behaviors [42]. The study explores the relationship between bike-sharing user activities in different event throughout using the services. The employed probabilistic model allows for information fetching on weekly activity patterns from check-in logs via Foursquare.

5) Ride-Hailing Services: Ride-hailing services has turned into mainstream methods for public to access transportation thanks to the advances of mobile computing and smart devices. The services facilitate many advantageous features including quick availability, on-demand, timesaving, flexible payment methods, privacy, and security. Previous travel behavior studies often work with datasets associated with Uber services due to its reputation in ride-hailing services. A transportation analysis study explored the rising popularity of Uber as mainstream method of transportation in the Greater Toronto and Hamilton Areas (GTHA) [43]. The study investigated the behavioral process of combined probabilistic decisions with consideration of process of formation and conditional semi-compensatory decision. However, the developed model does not indicate intricate comparison between Uber and private cars, public transport, and motorless vehicles. They also pointed that most young adults prefer Uber services due to its compatibility and flexibility for accommodating the trip. Nowadays, most ridehailing services embedded their facilities on smart devices and online platforms, providing users with quick access to transportation. TBM is used for supporting these facilities by facilitating timely demand availability, ride sharing organization, and travel punctuality. The current increasing preferences towards ride-hailing services indicates the increases of demand for automated vehicles in the future. Consequently, public adaptation towards mainstream ride-sourcing norm also triggers changes in transportation aspect like carpooling, biking, ride-hailing (Lyft, Uber), and micro-transit (Bridj, Chariot). Such instance of changes was recorded in a dataset comprising attributes like times, distance, and earning from rides, and travel behavior via social demographic interviews with 311 passengers [44].

\section{Travel Behavior Modeling Data Embedded Technologies}

Based on the survey, TBM are commonly embedded in many transportation technologies like autonomous vehicles, road sensing, GPS analytics, automatic fare, real-time traffic monitoring, big data, and smart vehicles. The role of TBM on empowering these technologies are discussed as follows.

1) Autonomous Vehicles: The premise of innovating autonomous vehicles (AV) is to allow drivers to perform other en-route tasks or activities apart from piloting the vehicle.
Powered by the integration of diffusion model and spatial travel demand model ingenuity, AV poses significant impact on future travel behavior. With mainstream adaptation especially in Germany and USA, AV are gaining attraction due to longterm travel duration and cost efficiency with least mobility impairment. TBM is essential in anticipating the travel behavior changes prompted by this adaptation and predicting its impact on travel demand. Consequently, policy recommendations to support the adaptation of $\mathrm{AV}$ and mitigating its negative consequences are crucial [45].

2) Global Positioning System (GPS): The travel pattern generated from GPS logs effectively reflects the daily movement of an individual and their travel behavior. A recent study observed and compared individual movement activities when inside and outside of their home using TBM, specifically C-Means (FCM) clustering algorithm [46]. The use of GPS also contributes towards generating many travel behavior data, that eventually leveraged for strategic urban planning. For instance, Dar es Salaam travel behavior data is generated using citizen GPS to help Tanzanian government in improving public transport policies and infrastructure [47]. TBM help processing these GPS data to generate insights on effective planning for street infrastructures, landmarks, and land use [48].

3) Road Sensing: In their study, $\mathrm{Su}$ et al. explores the utilization of road events to enhance the quality of road sensing system [49]. Road events like traffic jams, road accidents, potholes, bumps, and road signs further enrich the compatibility comparison of the suggested route. Subsequently, they proposed a low-cost crowd-sourcing data collection and integration system for detecting en-route risks and generating alternative routes. Safety risks and complications like slippery roads, pavement roughness, and travel regulation are considered based extracted information from crowd-sourced big data.

4) Automated Faring: In his work, Kang implemented scalable inference methods for analyzing the relationship between public transit system route and travel behavior [50]. The study focuses on the association between traveler's origin-destination choices with multi-modal travel environment. Two preference vectors and true OP-pairs were investigated using Automatic Fare Collection (AFC) system-type data (stop-level ODs). The investigation was possible after gathering and analyzing data for route choice with stochastic travel environment specifically for paying travel fare.

5) Real-Time Traffic Management: Such instance of TBM usage in real-time traffic management technologies is the transport model algorithm development by Wen and Chunming. The presented algorithm tracks and predicts real-time traffic dynamics based on agent-based transport model and independent observations of the trajectory of several hundred vehicles [51]. The study involves arrays of TBM technologies including big data, machine learning, and sensor networks for generating efficient travel time and fuel consumption.

6) Big Data Infrastructure: When working with big data, TBM is crucial to facilitate large scale spatial analysis on human mobility via movement monitoring. The analysis itself comprised of processing on many variables including preliminary analysis, path, trip purpose, origin-destination matrices, mode, path choices, and unresolved travel errors. Nevertheless, utilizing TBM with big data requires adaptation depending 
on the technique and methodologies used to process the data. Future TBM studies should work with larger data sample sizes to explore its scalability on facilitating accurate movement analysis [52].

7) Internet of Things (IoT): With mainstream use of travel route system, travel behavior data is generated in real-time using smart devices and IoT technologies. The data generated via this method often utilized to map travel routes of many travelers based on their point of interest. Using automated event detection, the system gathers and process information like visit duration and activities to generate the next best travel routes. Future TBM studies can develop algorithms that maps frequent tangible travel routes and personalize suggested route ranking.

\section{E. Travel Behavior Modeling Analysis}

In general, travel behavior studies examine and analyze human daily travel behavior and its impact to society development. This taxonomy classifies the spectrum of related behavior analysis into 4 classes: travel patterns, forecasting, behavior changes, and travel choices. Summarization of work examples on each type of behavior analysis are presented as follows.

1) Travel Patterns: Behavior analysis on travel patterns focuses on analyzing instances and patterns of travel activities over a period or duration. For example, Krueger et al. proposed an integrated model that facilitates travel pattern assessment with consideration of moral values, norms, modalities, and travel behavior [53]. The model enables rapid identification on any travel pattern modification with concurrent shifts in normative beliefs. However, further refinement of the model is crucial such as including latent parameter constructs like values, aims, and personal identity. In addition, the study plans refining the model to generate travel decisions in a comprehensive representation of social context. Another study highlighted the implications of recent travel behavior of working adults by observing their weekly travel movement. The observation involves working adults without young children in California districts, with the goal of increasing auto use and reducing active transit use. A recent study investigated on travel patterns and its relationship with household compositions in major cities for sustainable urban development [54]. Their findings reveal that insights from urban travel patterns helps city planners and policymakers in addressing societal barriers and sustainable traveling.

2) Travel Forecasting: Travel forecasting predicts predetermined outcomes based on existing event records to helps encounters future travel difficulties. A user can plan their travel accordingly based on the insights generated by travel forecasting system. In this context, the forecast of travel demand via user travel planning helps transportation scheduling and policy making. To generate simulated user travel planning, random forecast is utilized to generate ensembles of random decisions. A robust random forecast can predict and classify user travel preferences to further measure the model capacities and interpretability. Prediction of user travel preferences eventually contributes towards facilitating behavioral interpretation and pattern discovery in complex datasets.
3) Behavior Changes: The changes of dynamic between travel behavior and residential point revolves around three major changing conditions: relocation of travel purpose, residential location choice, and modification of residential environment [55]. Behavior changes in travel activities often characterized by factors or traits like travel satisfaction, travel attitudes, and travel behavior. Behavior changes also influenced by point of travel origin that is commonly home location, reflected towards the entirety of the travel plan. Maneuvering alternatives but unfamiliar routes during travel also triggers behavior changes depending on the travel locations.

4) Travel Choices: Predicting traveler's choice of destination based on emerging trends from previous travel behavior record is challenging. Researchers started to develop specific behavior modeling for assessing destination travel choices to allow predictions on multi-instances of destination choices. Inclusion of real market subdivision with regards to attitudinal parameters is crucial for model plausibility and statistical testing. The destination choice behavior modeling eventually leads towards enabling cross-function classification of other supporting parameters like estimation, goodness, and fitness. The entire process consists of two-stages: starting with common choice of functional destination, then adjustment based on rules allocation [56].

\section{F. Travel Behavior Modeling Models}

TBM observes travel demand conditions that are based on a variety of factors including location, place, and time. Travel demand and travel decision are interlinked due to travel decision dependency on variables like cost and transport mode. However, the true common factors between travel demand and travel decisions are typically the location, place, and destination. TBM models enables early prediction on travel demands but heavily reliant on given input like transport mode and precise location or destination. Such instances of TBM models are Individual TBM, Regret-based TBM, and Rethinking TBM, Short-term TBM. Derived from the survey, our taxonomy classifies TBM models into two major categories: facilitating travel decisions and facilitating travel demand.

1) Travel Decision: Travel decision is influenced by traveler's choices with cost, mode of transportation, time, weather, location, and route. The need for understanding traveler's choice bring forth the development of many variation of travel decision-based model. Generating ideal destination choices based on previous travel patterns are challenging especially with consideration of multitude of instances [56].

With increasing traffic congestion occurrence in many metropolitan cities, real-time traffic prediction and routing generation now becomes essential. Using provided information like ongoing routes, traffic density, and incident vicinity, TBM helps by generating optimal routes to destination. Such instance of TBM uses is in GPS routing that monitor trips via rapid route identification using probabilistic modeling. However, TBM implementation in GPS routing can still be improved in terms of accuracy with latest hardware fitting and en-route approach [8].

The goal of intra-household TBM is to facilitate accurate and effective travel decision-making based on daily intrahousehold interactions. Individual travel decisions often influ- 


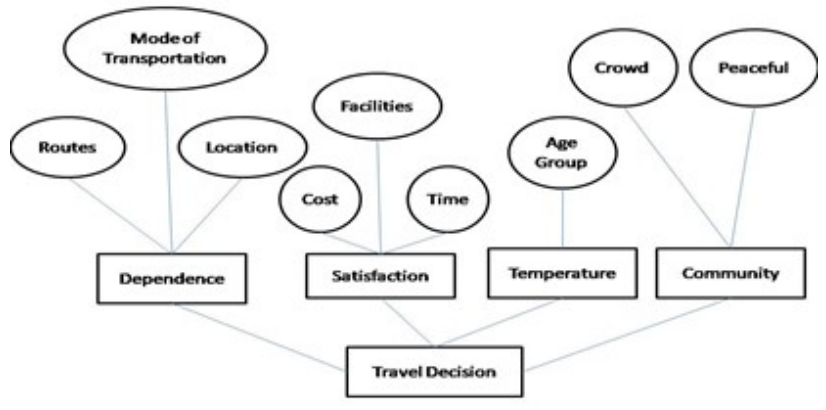

Fig. 3. Relational Variables in Travel Decisions Analysis.

enced by opinions, prior experience, and needs of household members or acquaintances. A study developed a method using this model for eliminating decision bias that restrained viable outcomes in accommodating intra-household travel necessity effectively. Bayesian-based multivariate spatial specification is implemented to allow individuals to take household readiness into account during generating travel decision.

Sense Place TBM was built to help identifying relations among the derives of six different variables representing a site. SPTBM is facing many problems with regards to data collection, planning practice, and modeling. A study used SPTBM with the aim to inspect the sense of a location's structure and measurement using survey data. There are three factors in sense of places that are fetched using the three-tiered structural equation model, which parallels estimated sense analysis factors. Sense analysis factors are mostly related to environment [10]. The integrated model of the system involves moral values, norms, modalities, and traveling behavior. This model can identify travel pattern modification during the time of travel in response to concurrent shifts in normative beliefs and behavior. The drawbacks of this model were addressed by bringing improvement in the framework in terms of more latent construct parameters like values, aims, and personal identity. Such approach allows for more comprehensive representation for travel decisions in a social context [53].

Markov Travel Model (MTM) focuses on travel decision concerning travel time, future foresting and individual preferences and behavior. Such TBMs that utilizes MTM concept are Class-Specific Mode Choice Model, Initialization Model, and Transition Model. These models would identify the evolution of preferences or modality styles. Possible enhancements to this study are based on preference dependencies for the individuals based on habits and framework improvements [11].

Another instance of travel decision model is Inferring Structural TBM that obtains socially optimal feedbacks on the utilization of a public-funded transportation infrastructure. This model leads to understanding the dynamics for governing the adoption of sustainable alternatives to driving with primary commute mode. Thus, a realistic decision-making approach is generated to improve the accuracy of travel decisions [12].

Fig. 3 summarizes the relational factors considered in travel decision analysis. Prime factors like dependence, satisfaction, temperature, and community possess additional sub-

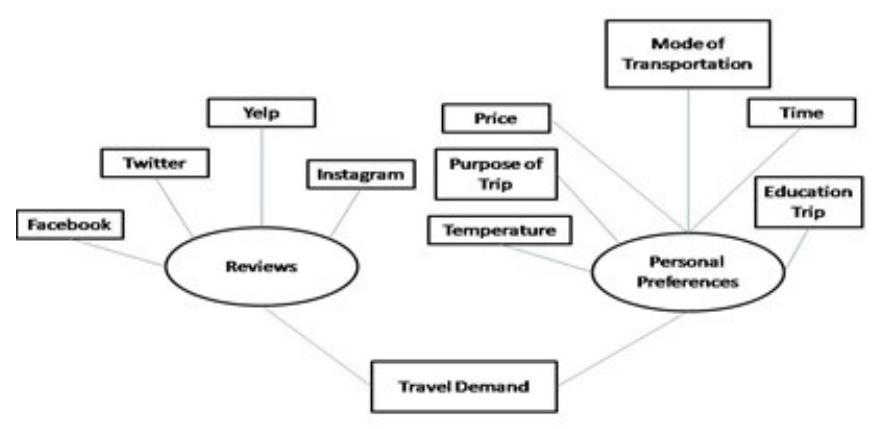

Fig. 4. Functional Model of Travel Behavior Modeling.

components. For instance, dependence has routes, mode of transportation and location. Routes may have different choices including clear route, rush route, less polluted route, or proper space for the vehicles. Mode of transportation has many options such as public transport, private vehicles, or bicycle walk, and location may have different choices like natural attraction, vacation planning, and business trip. Satisfaction factor depends on three parameters namely cost, facilities and time. Temperature has different effects on individuals depending on their age, young adults usually endure better compared to elders during winter. The community factor is dependent on whether the surrounding community emanating crowd or peaceful environment.

2) Travel Demand: Travel demand relies on the travel forecasting which depends upon varying parameters such as the most visited place, convenient hours, and seasons. There are several notable models that were developed to facilitate travel demand prediction analysis as discussed as follows.

Liang et al. developed Individual Travel Behavior (ITB) model focusing on identifying travel demand from public transport passengers travel activities. This model was built to analyze travel behavior individually for supporting feasible public transport implementation. Also known as the travel behavior graph-based model, ITB predicts individual graphs for passengers based on different public transport choices and demand, and provides an analysis of travel features with the help of customized public transport. There are several drawbacks of travel behavior graph-based models like fetching and scheduling the demand of research is higher with the optimization of the transport operating network justifiable on a large scale [14].

Regret-based choice models estimate travel demand on many levels and relate to various travel choice contexts. The models show outcomes for different traditional utilitybased models in comparison with behavioral interpretations. The objective of this type of model is to present regret based decision-making process to provide more quality and behavioral insights. Regret-based choice models are enhanced in terms of the framework by better disentanglement in various sources of heterogeneity [13].

Heterogeneous Travel Behavior (HTB) aims to facilitate trip generation and simulation. The HTB model skips the entire path to an activity-based model and can predict the 
TABLE I. Characteristics COMParison Between VARIOUS TBM Models.

\begin{tabular}{|c|c|c|c|c|}
\hline Reference & Model & Data Type & Data Size & Future Work \\
\hline [8] & Travel Decision & GPS & 260 individuals. & En-route prediction before and beginning of trip. \\
\hline [9] & Travel Decision & Survey & 67,000 trips, 3,500 individuals. & More accurate and effective travel decision. \\
\hline$[10]$ & Travel Decision & Survey & 719 individuals. & Enhancement using socio-demographic indicators. \\
\hline [9] & Travel Decision & Survey, mobile data & Not reported. & Transportation planning applications. \\
\hline$[53]$ & Travel Decision & Survey & 170 individuals. & Enhancement of framework. \\
\hline$[11]$ & Travel Decision & Interviews & $\begin{array}{l}\text { 1st wave (303), 2nd wave (286), 3rd wave } \\
\text { (258) individuals. }\end{array}$ & Enhancement of framework. \\
\hline [12] & Travel Decision & $\begin{array}{l}\text { Survey, Open Street } \\
\text { map data }\end{array}$ & Not reported. & Realistic decision-making approach. \\
\hline$[14]$ & Travel Demand & Smart card data & 6 passengers. & Automatic graph generation with larger scale computing. \\
\hline [13] & Travel Demand & Survey & 242 individuals. & $\begin{array}{l}\text { Significantly improves the performance of the random regret mini- } \\
\text { mization model to enhance the framework. }\end{array}$ \\
\hline$[16]$ & Travel Demand & $\begin{array}{l}\text { Glove embedding } \\
\text { vectors }\end{array}$ & $\begin{array}{l}6 \text { billion input word vectors from } 37 \text { million } \\
\text { texts. }\end{array}$ & $\begin{array}{l}\text { Stochastic nature of the algorithm that requires careful experimental } \\
\text { design. }\end{array}$ \\
\hline$[15]$ & Travel Demand & Survey & $\begin{array}{l}\text { Trips range from } 0 \text { to } 5 \text {, cross-classification } \\
\text { model with } 1.24 \text { trips. }\end{array}$ & Inclusion of additional variables for better accuracy rate. \\
\hline
\end{tabular}

development of travel demand with least computer processing time. HTB possess many advantages such as prediction of true travel speeds, mode-defined constants, and improved assignment results. Another model is Microscopic Trip Generation Module that maintain an operating model while improving the research and its design. However, there are some limitations in the model such as lack of accuracy, auto-ownership, and microscopic tour generation [15].

Fig. 4 summarizes the relational factors considered in travel demand analysis like reviews and personal references. Reviews are collected through social media. Personal preferences are more dependent to each traveler. Comparison of models in the term of data type, size, and future improvement suggestion is summarized in Table I.

The survey findings indicates that travel decision-based TBM future direction eventually leads towards precise en-route prediction. Precising the prediction requires investigation on crucial factors like pre-planning, transportation accommodation planning, and effective travel decision framework. The prospect of precise en-route prediction is viable and have lasting impact towards adapting TBM with realistic decisionmaking capabilities. Furthermore, existing travel demand based TBMs were largely constrained or limited by technical components and scalability aspects. These TBMs often hindered by computing limitation or handling large-scale data for automatic graph generation. Improving these components can increase the performance of applied random regret minimization model and framework enhancement. The inclusion of additional variables from datasets with greater size are recommended to enrich or enhance the model prediction accuracy.

\section{G. Travel Behavior Modeling Common Datasets}

Existing travel behavior studies have worked with many travel datasets from various sources ranging from open data, crowdsourcing, and conventional surveys. In the following discussions, we present the summarization of several notable travel behavior datasets based on our conducted survey.
1) Point of Interest Dataset: This dataset was shared to help researchers in finalizing trip purpose parameter for developing destination prediction algorithm with higher accuracy [8]. This dataset is generated via crowdsourcing Open Street Map with 260 participants, allowing their vehicle's location to be recorded for every 70 minutes. The dataset comprises attributes including trip time, weekdays, origin, purpose, user, trip number and most common destination visited. The original XML data was drawn via Geographic Information Systems (GIS) in the form of layers of files linked with GPS data. This dataset features relational travel information of roughly estimated 26,000,000 points of interest in American states of Maryland, Washington D.C., and Virginia.

2) London's Oyster Smart Card \& Survey Dataset: Since 2012, data generated by smart cards usage in London's transportation system has been used to create the dataset. There are two different smart cards and two different modes of transportation: tube and bus. 2.18 million journeys of 9708 passengers were recorded in the dataset. Furthermore, the smart cards dataset often paired with the London Travel Demand Survey dataset. The survey contains collected household information including household demographics, socioeconomic information, and travel-related information. The survey inquires common information like ID, age, manager, total number of owned vehicles, household income, working status, occupation, weekly work frequency, daily commuting distance, frequency of becoming driver or passengers [57].

3) Los Angeles Dataset: Using survey with 352 participants from 219 households over 2395 days, this dataset is generated to map individual daily travel activities. The dataset contains variables including information on total number of daily trips distributed by types of transport, demographic, employment status, household, owned vehicle, and annual income. Daily individual travel activities were recorded in weekly basis with exclusion of repetitive activities in daily basis [58].

4) Neighborhoods for Active Kids Dataset: This dataset is generated to help management to build and implement active school travel planning. The dataset includes communities 
TABLE II. RECOMMENDATIONS FOR OPTIMIZING USES ON COMMON TBM DATASETS.

\begin{tabular}{|c|c|c|}
\hline Datasets & Descriptions & Suggestion \\
\hline Point of Interests [8] & Destination prediction & $\begin{array}{l}\text { Develop more efficient algorithm and use age factor parameter in the } \\
\text { datasets for understating about age group destination prediction. }\end{array}$ \\
\hline $\begin{array}{l}\text { London's Oyster Card and } \\
\text { Survey [57] }\end{array}$ & Passenger's movement and travel demand forecasting & Develop an advisory system to be used during travel time. \\
\hline Los Angeles [58] & $\begin{array}{l}\text { Transportation professionals and policy makers consider shifting from } \\
\text { the conventional one-day approach toward a multi-day approach. }\end{array}$ & $\begin{array}{l}\text { Collaboration with IT industry and transportation industry for making } \\
\text { bridge between them. }\end{array}$ \\
\hline $\begin{array}{l}\text { Neighborhoods for active kids } \\
\text { [59] }\end{array}$ & $\begin{array}{l}\text { Independent mobility by providing child friendly social and safe } \\
\text { environment, traffic safety, and policies that promote local schools } \\
\text { and safe vehicle-free zones around schools. }\end{array}$ & $\begin{array}{l}\text { Development and enhancement of the ideas in terms of algorithm and } \\
\text { software. }\end{array}$ \\
\hline Kaunas City [60] & Travel time data collection & Develop a method for data security. \\
\hline Bandung City [61] & Measurement of transport demand & $\begin{array}{l}\text { Develop a strong policy for sustainable transportation in developing } \\
\text { countries. }\end{array}$ \\
\hline $\begin{array}{l}\text { National Household Travel } \\
\text { Survey (NHTS) - City of } \\
\text { Long Beach [62] }\end{array}$ & Trip destination prediction & Develop an algorithm for trip designation prediction. \\
\hline INRIX [63] & Traffic management & $\begin{array}{l}\text { Development of traffic management algorithm with increased accuracy } \\
\text { and efficiency. }\end{array}$ \\
\hline
\end{tabular}

to support independent mobility by providing traffic safety, social environment, local promotion school policy and safe vehicle areas around the children schools. Dataset variables consists of school travel mode and child characteristics such as age, gender, ethnicity, physical activity, child beliefs regarding traffic safety, neighborhood, independent mobility, and household characteristics. The household characteristics are education, employment, number of adults, number of children, car ownership, household beliefs, distance to the school, traffic safety, stranger danger, convenience, and social interaction. Social environment covers neighborhood, safety, neighborhood cohesion, neighborhood connection and other related factors such as residential density, street connectivity, high traffic exposure and low traffic exposure [59].

5) Kaunas City Dataset: This dataset original use is for facilitating state government to analyze car travel time pattern around the city. Developed using Python, a Geographic Information System (GIS) was created with the purpose of extracting, analyzing, and visualizing car travel data. The extracted data constitutes the dataset with information like coordinates of origin, coordinates of destination, mode of travel, date, and departure time [60].

6) Bandung Metropolitan City Dataset: Bandung Metropolitan City dataset recorded traveler's check-in behavior, comprising enriched insights on understand traveler's preferences. Personal verification is required including user ID, gender, date, time, and geo-location (longitude and latitude), and address. The attributes contained in this dataset is closely similar to Shanghai dataset including total number of check-ins, total number of processed check-ins, and total number of users distributed by gender [61].

7) National Household Travel Survey (NTHS) - City of Long Beach Dataset: The NHTS dataset is generated via conventional survey conducted in the city of Long Beach, California. The survey was conducted to gather consensus on environmental factors at trip destinations that affect nonmotorized travel behavior in the area. The national household travel survey was conducted between 2008 to 2009 comprising information on motorized users, area density, diversity, and design at destinations that significantly affect mode choice decisions [62].

8) INRIX Dataset: The Floating Car Data (FCD) of Netherlands focuses on traffic information, traffic management and automated vehicles. The INRIX dataset recorded travel time of persons and goods from place of origin to destination (O-D) and hours of delay [63].

Based on the condition of presented datasets, several recommendations are suggested to help future TBM studies in optimizing uses of these datasets. The suggested recommendations for each dataset are summarized in Table II.

\section{Research Challenges in Travel Behavior MODELING APPLICATIONS}

TBM acts as simulator and generate decision-making insights and trends for travel decision and travel demand analysis. The output insights are used to strategize effective land use, vehicle sales, infrastructure investment and planning, and service improvement. Despite the many advantages, leveraging TBM optimally are challenging in many aspects including limitation of enabler technologies, data sources, and collection methods. The classifications based on our taxonomy reveals several emerging trends and challenges in utilizing TBM. We derived these challenges by analyzing available common parameters in recent TBM works and classified them based on elements in our TBM framework in Fig. 1. The research challenges are deliberately discussed as follows.

\section{A. Technological Limitation}

Many studies on TBM methods and technologies have been carried out over the years. However, these studies also encounter limitations like model inaccuracy or inefficiency, partial framework, transport-dependent planning, unrealistic decision manner, and inconstant socio-demographic. The survey results indicates that future work on TBM eventually heading towards the application of generalized probabilistic models. Future TBM possibly requires intricate programming development of behavior models for processing massive mobile apps data with increased efficiency and accuracy. 
TABLE III. Summarization on Challenges, ReCommendations, AND Future DiRECTIONS For TBM.

\begin{tabular}{|c|c|c|c|}
\hline Challenges & Objectives & Recommendations & Future Directions \\
\hline Behavior Modeling & $\begin{array}{l}\text { - Predicting individual travel behavior } \\
\text { - Individual TBM Graph }\end{array}$ & $\begin{array}{l}\text { - Develop methods for suspicious behavior prediction } \\
\text { - Advanced case detection on sudden behavior change }\end{array}$ & $\begin{array}{l}\text { - Travel demand prediction } \\
\text { - Automatic behavior graph generation }\end{array}$ \\
\hline Destination Prediction & $\begin{array}{l}\text { - Trip prediction purpose } \\
\text { - Predicting destination location } \\
\text { - Advanced pre-input route generation }\end{array}$ & $\begin{array}{l}\text { - Long-term behavior prediction } \\
\text { - Integrating emergency alert service } \\
\text { - Remote prediction without internet dependencies }\end{array}$ & $\begin{array}{l}\text { - En-route prediction of destination } \\
\text { - Increasing prediction accuracy rate }\end{array}$ \\
\hline Policy & $\begin{array}{l}\text { - Feedback investigation } \\
\text { - Travel planning environment } \\
\text { - Schedule composition and activity duration }\end{array}$ & $\begin{array}{l}\text { - False feedback prediction } \\
\text { - Smart cities development support } \\
\text { - Policy proposals }\end{array}$ & $\begin{array}{l}\text { - Decision-making to match real-world } \\
\text { behavior }\end{array}$ \\
\hline $\begin{array}{l}\text { Expensive Autonomous } \\
\text { Vehicles }\end{array}$ & $\begin{array}{l}\text { - Reducing public transportation } \\
\text { - Ride sharing network promotion }\end{array}$ & $\begin{array}{l}\text { - Public adaptation } \\
\text { - Low-cost maintenance }\end{array}$ & $\begin{array}{l}\text { - Low-cost development of autonomous } \\
\text { vehicles }\end{array}$ \\
\hline
\end{tabular}

TBM is used in many instances including travel management, traffic management, medical access, healthcare planning, and sustainable urban planning. TBM can generate timely decision-making insights in case of additional input like dietary preferences, emergency events, or destination changes. Despite the many usage instances, TBM is in fact heavily used in many processes of traffic management and planning. Several methods employ TBM to facilitate traffic management like spatiotemporal monitoring [64], closed-circuit TV camera [65], smart vehicle technology [66], traffic control [67], and movement behavior [68].

\section{B. Quality of Generated Data for Modeling}

TBM output relies on the dataset quality it works with, depending on the reliability of its data source and collection method. Albeit the dataset quality often reduced due to data privacy measures, these measures are necessary to protect data sources and users from external threat. The prospect of combining TBM with effective user mobile data access control and monitoring are considered viable for future TBM. Accurate direct access can further enrich generated insights for user travel planning, preferences, routes, and safety using realtime information. Moreover, data quality from conventional surveys like questionnaire and interview are diminished by high inaccuracy rate due to invalid feedback and lack of verification.

Nowadays, social media usage serves as the prime data sources for analyzing travel behavior and experiences. Social media data holds rich information like user posts, opinions, or discussions on their travel experiences. However, real-time social media datasets are not available for the public due to provider's policies [31]. For instance, users can retrieve data generated by Google Maps via application programming, however, certain personal information like travel speed, gender, vehicles, and cost may be redacted. Therefore, mutual collaborations between researchers and social media providers in research should be intensified productively to fully utilize the potential of TBM.

Apart from social media, the mainstream use of ride-hailing services also generates many useful datasets for travel behavior studies. Travel datasets generated by ride-hailing services features unique perspectives on travel behavior like on-demand services, quick booking, and time-critical traveling. However, similar to policies restriction of social media, ride services like Uber and Grab does not share their data to the public [44].
Due to the nature of the business, corporate reputation is of utmost important that any allegation of misconduct or misuse are devastatingly damaging. Albeit the unique characteristics of the ride services datasets, its data quality is also affected by many external factors and dependencies. External factors like internet connectivity, unavailability of drivers during holidays, and apps usability eventually determines the quality of the generated datasets.

\section{OPEn ISSUES AND OPPORTUNITIES FOR TRAVEL BEHAVIOR MODELING}

This section discusses and summarize the identified open issues emerging from the research challenges found in the survey. Despite the recent advances that attempted to address the challenges, TBM itself revolves around human user which is a complex system. Considering the complexities and difficulties in understanding human behavior, future TBM research should focuses on personalization aspect to address the remaining issues. The remaining open issues are summarized in Table III and discussed as follows.

1) Quality Behavior Modeling: Modeling travel behavior duration is crucial in facilitating individual travel behavior prediction and model graph generation for supporting analysis on travel decisions and demands. The modeling procedure generates automatic travel behavior graph using computing [14] and models the behavior prediction [13]. The constant need for accurate rapid event prediction in person travelling activity, particularly if the behavior has become routine, is a persistent problem in behavior modelling. Therefore, methods for understanding sudden behavior changes in the middle of traveling trip is necessary to anticipate risks and maneuver. Future studies also can concentrate on enhancing travel activity data collection approaches and channels for generating insights on encouraging quality personal travel behavior.

2) Real-Time Destination Prediction: Destination prediction remains as one of the major issues in TBM that is influenced by many goal variables like en-route trip, destination location, and advance travel route information. Modeling destination prediction often constrained by dependencies on high accuracy rate of en-route prediction of destination [8]. In addition, the accuracy rate is often affected by internet connectivity especially for facilitating long-term travels or remote locations. To address this issue, travel application should provide comprehensive planning mode that generate insights by taking account trip preparation. This mode also should actively 
identify mapping point with available communication access and store the coordinates for offline viewing. Furthermore, future studies should focus on the scope of facilitating realtime route changes with consideration of cost, duration, and safety.

3) Intelligent Travel Policies: Existing policies focuses on intelligent travel decisions including feedback investigation, environmental impact, schedule composition planning, activity duration, and emergency readiness. However, there are limited policy guidelines for encouraging quality travel decisionmaking that matches real-world travel behavior [12]. The increased possibility of faulty travel decision-making increases the likelihood of ensuing problem and its negative impact. Therefore, methods to differentiate between valid feedback and invalid feedback is critical to eliminate any change outlier during behavior modeling.

4) Cost-Efficient Autonomous Vehicles: Adaptation and usability of autonomous vehicles remains the main issue for anticipating future travel behavior and building ride-sharing practices. This issue stemmed from the persisting high cost of adopting or implementing autonomous vehicles in transportation ecosystem. Therefore, the development of low-cost autonomous vehicles is paramount for large-scale public adaptation into mainstream transportation [4], [5], [6].

\section{CONCLUSions AND Future Work}

In this paper, we presented overview insights on recent advances in TBM encompassing modeling classifications, emerging challenges, and arising opportunities. Leveraging insights from travel demand and travel decision analysis, TBM enables the prediction of future possibilities of travel scenarios. A taxonomy of travel behavior modeling along with the characterization and summarization of works on each classification were presented. Based on the survey, recent advances in TBM are distinctly classified based on algorithms, data sources, technologies, travel behavior, models, and datasets. Despite its heavy uses in facilitating transportation sectors, TBM also indirectly supports other sectors like tourism, marketing, and urban planning. Based on the reported challenges in recent TBM advances, we learn that model inaccuracy and inefficiency issues persist and largely constrained by current technological limitation and dataset qualities. The prospect of improving TBM via large scale integration and crowdsourcing are considered viable due to increased likelihood for better accuracy and efficiency. Based on the survey, we identified several notable issues and opportunities arising from recent TBM advances revolving aspects like behavior modeling, destination prediction, policy, and expensive autonomous vehicles. Our findings envision that future travel behavior are expected to change drastically due to many substantial aspect evolutions. Therefore, TBM is expected to play significant roles in realizing these visions. Researchers and industry analysts can investigate the TBM open issues and research opportunities highlighted in this survey for future undertaking. Future studies can also leverage the proposed taxonomy and identified challenges to enrich understanding on TBM for improvement purposes.

\section{REFERENCES}

[1] Z. Kan, L. Tang, M.-P. Kwan, C. Ren, D. Liu, and Q. Li, "Traffic congestion analysis at the turn level using Taxis' GPS trajectory data,"
Computers, Environment and Urban Systems, vol. 74, pp. 229-243, 2019.

[2] H. M. Amer, H. Al-Kashoash, M. Hawes, M. Chaqfeh, A. Kemp, and L. Mihaylova, "Centralized simulated annealing for alleviating vehicular congestion in smart cities," Technological Forecasting and Social Change, vol. 142, pp. 235-248, 2019.

[3] A. Soteropoulos, M. Berger, and F. Ciari, "Impacts of automated vehicles on travel behaviour and land use: an international review of modelling studies," Transport Reviews, vol. 39, no. 1, pp. 29-49, 2019.

[4] J. Bischoff and M. Maciejewski, "Simulation of city-wide replacement of private cars with autonomous taxis in Berlin," Procedia Computer Science, vol. 83, pp. 237-244, 2016.

[5] L. M. Martinez and J. M. Viegas, "Assessing the impacts of deploying a shared self-driving urban mobility system: An agent-based model applied to the city of Lisbon, Portugal," International Journal of Transportation Science and Technology, vol. 6, no. 1, pp. 13-27, 2017.

[6] W. Zhang, S. Guhathakurta, and E. B. Khalil, "The impact of private autonomous vehicles on vehicle ownership and unoccupied VMT generation," Transportation Research Part C: Emerging Technologies, vol. 90, pp. 156-165, 2018.

[7] J. M. Dargay and M. Hanly, "The impact of land use patterns on travel behaviour," in European Transport Conference, France, 2003.

[8] C. M. Krause and L. Zhang, "Short-term travel behavior prediction with GPS, land use, and point of interest data," Transportation Research Part B: Methodological, vol. 123, pp. 349-361, 2019.

[9] C. Kim and O. Parent, "Modeling individual travel behaviors based on intra-household interactions," Regional Science and Urban Economics, vol. 57, pp. 1-11, 2016.

[10] K. Deutsch, S. Y. Yoon, and K. Goulias, "Modeling travel behavior and sense of place using a structural equation model," Journal of Transport Geography, vol. 28, pp. 155-163, 2013.

[11] F. E. Zarwi, A. Vij, and J. Walker, "Modeling and forecasting the evolution of preferences over time: A hidden Markov model of travel behavior," arXiv preprint arXiv:1707.09133, 2017.

[12] S. Feygin, "Inferring Structural Models of Travel Behavior: An Inverse Reinforcement Learning Approach," Ph.D. dissertation, 2018.

[13] S. Jang, "Regret-based travel behavior modeling: an extended framework," Ph.D. dissertation, 2018.

[14] Q. Liang, J. Weng, W. Zhou, S. B. Santamaria, J. Ma, and J. Rong, "Individual travel behavior modeling of public transport passenger based on graph construction," Journal of Advanced Transportation, vol. 2018, 2018.

[15] R. Moeckel, L. Huntsinger, and R. Donnelly, "From macro to microscopic trip generation: representing heterogeneous travel behavior," The Open Transportation Journal, vol. 11, no. 1, 2017.

[16] F. C. Pereira, "Rethinking travel behavior modeling representations through embeddings," arXiv preprint arXiv:1909.00154, 2019.

[17] J. C. Willems, "Models for dynamics," in Dynamics reported. Springer, 1989, pp. 171-269.

[18] Q. Qi, T. Weise, and B. Li, "Optimization algorithm behavior modeling: A study on the traveling salesman problem," in 2018 Tenth International Conference on Advanced Computational Intelligence (ICACI). IEEE, 2018, pp. 861-866.

[19] M. Wong and B. Farooq, "A bi-partite generative model framework for analyzing and simulating large scale multiple discrete-continuous travel behaviour data," Transportation Research Part C: Emerging Technologies, vol. 110, pp. 247-268, 2020.

[20] D. Nam, H. Kim, J. Cho, and R. Jayakrishnan, "A model based on deep learning for predicting travel mode choice," Tech. Rep., 2017.

[21] A. N. P. Koushik, M. Manoj, and N. Nezamuddin, "Machine learning applications in activity-travel behaviour research: a review," Transport Reviews, vol. 40, no. 3, pp. 288-311, 2020.

[22] G. Sierpiński, M. Staniek, and I. Celiński, "Travel behavior profiling using a trip planner," Transportation Research Procedia, vol. 14, pp. 1743-1752, 2016.

[23] M. Berger and M. Platzer, "Field evaluation of the smartphone-based travel behaviour data collection app "SmartMo"," Transportation Research Procedia, vol. 11, pp. 263-279, 2015. 
[24] S. Jamal and M. A. Habib, "Smartphone and daily travel: How the use of smartphone applications affect travel decisions," Sustainable Cities and Society, vol. 53, p. 101939, 2020.

[25] S. F. Dias and V. A. Afonso, "Innovative Business Models in Tourism and Hospitality: Going Mobile?" in Strategic Business Models to Support Demand, Supply, and Destination Management in the Tourism and Hospitality Industry. IGI Global, 2020, pp. 164-184.

[26] M. Christian, "Mobile Application Development in the Tourism Industry and its Impact on On-Site Travel Behavior," Modul Vienna University, 2015.

[27] Y. Guo and S. Peeta, "Impacts of personalized accessibility information on residential location choice and travel behavior," Travel Behaviour and Society, vol. 19, pp. 99-111, 2020.

[28] B. G. Menon and B. Mahanty, "Modeling Indian four-wheeler commuters' travel behavior concerning fuel efficiency improvement policy;" Travel Behaviour and Society, vol. 4, pp. 11-21, 2016.

[29] N. S. Daisy, M. H. Hafezi, L. Liu, and H. Millward, "Understanding and modeling the activity-travel behavior of university commuters at a large Canadian university," Journal of Urban Planning and Development, vol. 144, no. 2, p. 4018006, 2018

[30] M. Rizwan and W. Wan, "Big data analysis to observe check-in behavior using location-based social media data," Information, vol. 9, no. 10, p. 257, 2018.

[31] S. A. Golder and M. W. Macy, "Digital footprints: Opportunities and challenges for online social research," Annual Review of Sociology, vol. 40, pp. 129-152, 2014.

[32] T. H. Rashidi, A. Abbasi, M. Maghrebi, S. Hasan, and T. S. Waller, "Exploring the capacity of social media data for modelling travel behaviour: Opportunities and challenges," Transportation Research Part C: Emerging Technologies, vol. 75, pp. 197-211, 2017.

[33] H. Cramer, M. Rost, and L. E. Holmquist, "Performing a checkin: emerging practices, norms and'conflicts' in location-sharing using foursquare," in Proceedings of the 13th International Conference on Human Computer Interaction with Mobile Devices and Services, 2011, pp. 57-66.

[34] A. P. Timalsena, A. Marsani, and H. Tiwari, "Impact of Traffic Bottleneck on Urban Road: A Case Study of Maitighar-Tinkune Road Section," in Proceedings of IOE Graduate Conference, vol. 5, 2017.

[35] J. Chang and E. Sun, "Location3: How users share and respond to location-based data on social," in Proceedings of the International AAAI Conference on Web and Social Media, vol. 5, no. 1, 2011.

[36] V. Jadhav, S. Raman, N. Patwa, K. Moorthy, and J. Pathrose, "Impact of Facebook on leisure travel behavior of Singapore residents," International Journal of Tourism Cities, 2018.

[37] M.-L. Zhang and Z.-H. Zhou, "A review on multi-label learning algorithms," IEEE transactions on knowledge and data engineering, vol. 26, no. 8, pp. 1819-1837, 2013.

[38] J. H. Lee, S. Gao, and K. G. Goulias, "Can Twitter data be used to validate travel demand models?" in 14th International Conference on Travel Behaviour Research, 2015.

[39] J. H. Lee, A. W. Davis, S. Y. Yoon, and K. G. Goulias, "Activity space estimation with longitudinal observations of social media data," Transportation, vol. 43, no. 6, pp. 955-977, 2016.

[40] E. Parra-López, J. Bulchand-Gidumal, D. Gutiérrez-Taño, and R. DíazArmas, "Intentions to use social media in organizing and taking vacation trips," Computers in Human Behavior, vol. 27, no. 2, pp. 640-654, 2011.

[41] S. Hasan and S. V. Ukkusuri, "Social contagion process in informal warning networks to understand evacuation timing behavior," Journal of Public Health Management and Practice, vol. 19, pp. S68-S69, 2013

[42] C. Coffey and A. Pozdnoukhov, "Temporal decomposition and semantic enrichment of mobility flows," in Proceedings of the 6th ACM SIGSPATIAL international workshop on location-based social networks, 2013, pp. 34-43.

[43] K. N. Habib, "Mode choice modelling for hailable rides: An investigation of the competition of Uber with other modes by using an integrated non-compensatory choice model with probabilistic choice set formation," Transportation Research Part A: Policy and Practice, vol. 129, pp. 205-216, 2019.

[44] A. Henao, Impacts of Ridesourcing-Lyft and Uber-on Transportation Including VMT, Mode Replacement, Parking, and Travel Behavior University of Colorado at Denver, 2017.
[45] L. Kröger, T. Kuhnimhof, and S. Trommer, "Does context matter? A comparative study modelling autonomous vehicle impact on travel behaviour for Germany and the USA," Transportation Research Part A: Policy and Practice, vol. 122, pp. 146-161, 2019.

[46] H. Millward, M. H. Hafezi, and N. S. Daisy, "Activity travel of population segments grouped by daily time-use: GPS tracking in Halifax, Canada," Travel Behaviour and Society, vol. 16, pp. 161-170, 2019.

[47] L. Joseph, A. Neven, K. Martens, O. Kweka, G. Wets, and D. Janssens, "Measuring individuals' travel behaviour by use of a GPS-based smartphone application in Dar es Salaam, Tanzania," Journal of Transport Geography, vol. 88, p. 102477, 2020.

[48] G. Lue and E. J. Miller, "Estimating a Toronto pedestrian route choice model using smartphone GPS data," Travel Behaviour and Society, vol. 14, pp. 34-42, 2019.

[49] L. Su, J. Gao, and Q. He, "Towards Quality-Aware Big Data Integration for Crowdsourced Road Sensing System,” 2017.

[50] J. E. J. Kang, "Inferring Origin-Destination Demand and UtilityBased Travel Preferences in Multi-Modal Travel Environment Using Automatic Fare Collection Data," Ph.D. dissertation, 2016.

[51] D. Wen and Q. Chunming, "Variational Inference for Agent-Based Models with Applications to Achieve Fuel Economy," 2017.

[52] C. Chen, J. Ma, Y. Susilo, Y. Liu, and M. Wang, "The promises of big data and small data for travel behavior (aka human mobility) analysis," Transportation Research Part C: Emerging Technologies, vol. 68, pp. 285-299, 2016.

[53] R. Krueger, A. Vij, and T. H. Rashidi, "Normative beliefs and modality styles: a latent class and latent variable model of travel behaviour," Transportation, vol. 45, no. 3, pp. 789-825, 2018.

[54] S. Chakrabarti and K. Joh, "The effect of parenthood on travel behavior: Evidence from the California Household Travel Survey," Transportation Research Part A: Policy and Practice, vol. 120, pp. 101-115, 2019.

[55] B. Devos and C. Werner, Culture and cultural politics under Reza Shah: The Pahlavi state, new bourgeoisie and the creation of a modern society in Iran. Routledge, 2013.

[56] J. A. Ansah, "Destination choice set definition in travel behaviour modelling," Transportation Research, vol. 11, no. 2, pp. 127-140, 1977.

[57] Y. Zhang, T. Cheng, and N. S. Aslam, "Exploring the Relationship Between Travel Pattern and Social-Demographics using Smart Card Data and Household Survey," International Archives of the Photogrammetry, Remote Sensing \& Spatial Information Sciences, 2019.

[58] C. Li, L. Hou, B. Y. Sharma, H. Li, C. Chen, Y. Li, X. Zhao, H. Huang, Z. Cai, and H. Chen, "Developing a new intelligent system for the diagnosis of tuberculous pleural effusion," Computer Methods and Programs in Biomedicine, vol. 153, pp. 211-225, 2018.

[59] E. Ikeda, E. Hinckson, K. Witten, and M. Smith, "Assessment of direct and indirect associations between children active school travel and environmental, household and child factors using structural equation modelling," International Journal of Behavioral Nutrition and Physical Activity, vol. 16, no. 1, p. 32, 2019.

[60] V. Dumbliauskas, V. Grigonis, and A. Barauskas, "Application of Google-based data for travel time analysis: Kaunas city case study," Promet-Traffic\&Transportation, vol. 29, no. 6, pp. 613-621, 2017.

[61] T. B. Joewono, A. K. M. Tarigan, and M. Rizki, "Segmentation, classification, and determinants of in-store shopping activity and travel behaviour in the digitalisation era: The context of a developing country," Sustainability, vol. 11, no. 6, p. 1591, 2019.

[62] D. Kim, J. Park, and A. Hong, "The role of destination's built environment on nonmotorized travel behavior: A case of Long Beach, california," Journal of Planning Education and Research, vol. 38, no. 2, pp. 152-166, 2018.

[63] H. van der Loopa, M. Kouwenhovenb, P. van Bekkumc, and H. Meursc, "Validation and usability of floating car data for transportation policy research," 2018.

[64] Y. Xu, Q.-J. Kong, and Y. Liu, "Short-term traffic volume prediction using classification and regression trees," in 2013 IEEE Intelligent Vehicles Symposium (IV). IEEE, 2013, pp. 493-498.

[65] V. Jain, A. Sharma, and L. Subramanian, "Road traffic congestion in the developing world," in Proceedings of the 2nd ACM Symposium on Computing for Development, 2012, pp. 1-10. 
[66] X. Chang, H. Li, J. Rong, Z. Huang, X. Chen, and Y. Zhang, "Effects of on-board unit on driving behavior in connected vehicle traffic flow," Journal of Advanced Transportation, vol. 2019, 2019.

[67] S. B. Raheem, W. A. Olawoore, D. P. Olagunju, and E. M. Adeokun, "The cause, effect and possible solution to traffic congestion on Nigeria road (a case study of Basorun-Akobo road, Oyo state)," International
Journal of Engineering Science Invention, vol. 4, no. 9, pp. 10-14, 2015.

[68] Z. Li and J. Huang, "How to mitigate traffic congestion based on improved ant colony algorithm: A case study of a congested old area of a metropolis," Sustainability, vol. 11, no. 4, p. 1140, 2019. 